\title{
Global Existence of Solutions of the Navier-Stokes- Maxwell System in Besov Spaces
}

\author{
Haifeng Bai and $\mathrm{Li} \mathrm{Li}^{*}$ \\ Department of Mathematics, Harbin Institute of Technology, Harbin 150001, \\ P.R. China.
}

Received June 17, 2018; Accepted November 12, 2018

\begin{abstract}
The motion of hydro-magnetic fluid can be described by Navier-StokesMaxwell system. In this paper, we prove global existence and uniqueness for the solutions of Navier-Stokes-Maxwell system in 3 dimensional space for small data.
\end{abstract}

AMS subject classifications: 35Q30, 35Q35, 76D03, 76D05

Key words: Navier-Stokes-Maxwell system, Besov space, existence, uniqueness, Littlewood-Play operator.

\section{Introduction}

Consider the Navier-Stokes-Maxwell (NSM) system in $\mathbb{R}^{3}$

$$
\left\{\begin{array}{l}
u_{t}-\triangle u+u \cdot \nabla u+\nabla p=j \times B, \\
E_{t}-\nabla \times B=-j, \\
B_{t}+\nabla \times E=0 \\
\operatorname{div} u=0, \quad \operatorname{div} B=0, \\
u(x, 0)=u_{0}(x), \quad B(x, 0)=B_{0}(x), \quad E(x, 0)=E_{0}(x),
\end{array}\right.
$$

where $u$ is the velocity field of the hydro-magnetic fluid, $E$ and $B$ are electrical and magnetic fields respectively, $p$ is the pressure, $j$ is the current density, which is governed by Ohm's law

$$
j=\sigma(E+u \times B) .
$$

The constant $\sigma$ is resistance, $u_{0}, B_{0}, E_{0}$ are initial values. Without loss of generality, we can choose the electric resistivity $\sigma=1$ hereinafter. The aim of this paper is to prove the existence of mild solutions of (1.1) in Besov spaces.

${ }^{*}$ Corresponding author. Email addresses: baihfqing@hit.edu.cn (H. Bai), leeeee@it.edu.cn (L. Li) 
Definition 1.1. We say that $(u, E, B)$ is a mild solution of (1.1) if it satisfies the integral equations

$$
\begin{aligned}
& u(t)=e^{t \triangle} u_{0}-\int_{0}^{t} e^{\left(t-t^{\prime}\right) \triangle} \mathcal{P}\left(\nabla(u \otimes u)\left(t^{\prime}\right)-j \times B\right) \mathrm{d} t^{\prime}, \\
& \left(\begin{array}{l}
E \\
B
\end{array}\right)=e^{t L}\left(\begin{array}{c}
E_{0} \\
B_{0}
\end{array}\right)-\int_{0}^{t} e^{\left(t-t^{\prime}\right) L}\left(\begin{array}{c}
v \times B \\
0
\end{array}\right) \mathrm{d} t^{\prime},
\end{aligned}
$$

where $\mathcal{P}: L^{2} \rightarrow\left\{u \in L^{2}: \operatorname{div} u=0\right\}$ is Leray's projection operator

$$
\mathcal{P}(u)_{j}(x)=\frac{1}{(2 \pi)^{n / 2}} \sum_{k} \int_{\mathbb{R}^{n}}\left(\delta_{j k}-\frac{\xi_{j} \xi_{k}}{|\xi|^{2}} \hat{u}_{k}(\xi)\right) e^{i \xi \cdot x} \mathrm{~d} \xi,
$$

and $L$ is the matrix operator

$$
L=\left(\begin{array}{cc}
-I & \text { curl } \\
- \text { curl } & 0
\end{array}\right)
$$

The motion of charged viscous fluid can be described by the Navier-Stokes-Maxwell equations, which was derived based on the classical Newtonian dynamics and Maxwell's electromagnetism theory.

In 2010, Masmoudi [16] obtained the global existence of the regular solutions of the Navier-Stokes-Maxwell system in $\mathbb{R}^{2}$, and proved the exponential growth rate. Duan [8] derived the Navier-Stokes-Maxwell system from the Vlasov-Maxwell-Boltzmann system by using the macro-micro decomposition of Liu, et al. [15], he also prove the global existence of solutions for the Cauchy problem of the compressible NSM system and analyzed the large time behavior of the solutoins. In 2015, Germain et al. [10] study the well-posedness of mild solutions of the NSM system. Chen and Jügel [6] analyzed the global existence of the solutions for the incompressible Navier-Stokes-Maxwell-Stefan system and proved that the global solution converges to the homogeneous steady state in time. Yang and Wang [21] proved that the regular solution of the compressible NSM system converges to the regular solution of the incompressible NSM system by using a new energy functional. In 2016, Tan and Tong [19] studied the global existence and large time behavior of the compressible Navier-Stokes-Maxwell system in $\mathbb{R}^{3}$ with linear damping. Liu and $\mathrm{Su}$ [14] obtained global existence near the constant steady states for the non-isentropic Navier-Stokes-Maxwell system. Ibrahim et al. [18] established the existence and asymptotic stability for the time periodic small solutions of the incompressible Navier-Stokes-Maxwell system in the whole $\mathbb{R}^{3}$.

The study on the weak solutions of partial differential equations usually proceed in suitable function spaces, Sobolev spaces for examples. Besov spaces can be viewed as an extension of the classical Sobolev spaces. So the well-posedness of the solutions in Besov spaces has theoretical value for mathematicians, especially in the case when the well-posedness is unknown in classical Sobolev spaces. In 1992, Kobayashi and Muramatu [12] proved the local existence, uniqueness and regularity of the solutions for 
the Cauchy problem of the unsteady Navier-Stokes equations in Besov spaces. Kozono et al. [13] proved the local existence for Navier-Stokes equations with initial data lying in Besov spaces, and established the extension criterion on local solutions based on the vorticity in homogeneous Besov spaces. In 2012, Bae et al. [1] proved the analyticity of solutions in Besov spaces by using Gevrey estimates in critical Besov spaces, and obtained the decay estimates. Chikami and Danchin [7] achieved the well-posedness of solutions for the complete compressible Navier-Stokes equations in the critical Besov spaces. In 2016, Chemin et al. [5] proved the local existence of solutions of the viscous MHD system in the whole space $\mathbb{R}^{n}, n=2,3$, and obtained uniqueness in 3D case. Fefferman et al. [9] obtained the local existence and uniqueness of the solutions for the non-resistive magnetohydrodynamics equation in the optimal Sobolev spaces. In particular, Ibrahim and Keraani [11] proved the existence and uniqueness of solutions to the Navier-StokesMaxwell system in a product space of Besov and Hilbert spaces. The present work can be viewed as a modification of theirs.

Our main result can be stated as follows.

Theorem 1.1. Set

$$
\mathbf{X}:=\dot{B}_{2,1}^{1 / 2}\left(\mathbb{R}^{3}\right) \times \dot{B}_{2,1}^{1 / 2}\left(\mathbb{R}^{3}\right) \times \dot{B}_{2,1}^{1 / 2}\left(\mathbb{R}^{3}\right) .
$$

There exists a small constant $\delta>0$ such that for any $\left(u_{0}, E_{0}, B_{0}\right) \in \mathbf{X}$ and $\left\|\left(u_{0}, E_{0}, B_{0}\right)\right\|_{\mathbf{X}}<\delta$, there exists a unique global solution $(u, E, B)$ of $(1.1)$ and

$$
u \in C\left(\mathbb{R}^{+} ; \dot{B}_{2,1}^{1 / 2}\right) \cap \tilde{L}^{2}\left(\mathbb{R}^{+} ; \dot{B}_{2,1}^{3 / 2}\right), \quad E \in \tilde{L}^{\infty}\left(\mathbb{R}^{+} ; \dot{B}_{2,1}^{1 / 2}\right), \quad B \in \tilde{L}^{\infty}\left(\mathbb{R}^{+} ; \dot{B}_{2,1}^{1 / 2}\right) .
$$

The organization of the paper is as follows. In next section, we will recall the definitions of Besov spaces, and the embeddings and fundamental inequalities in Besov spaces. In Section 3, the a priori estimates of the solutions to an approximation scheme is obtained. The proof of Theorem 1.1 is given in Section 4 .

\section{Preliminaries}

\subsection{Function spaces}

Besov spaces can be viewed as an extension of Sobolev spaces. For completeness of the description, we first review the definitions and properties of Besov spaces. See more details in for example $[3,17,20]$.

Denote $\mathcal{S}$ the test function spaces,

$$
\mathcal{S}\left(\mathbb{R}^{n}\right):=\left\{f \in C^{\infty}\left(\mathbb{R}^{n}\right)\left|\sup _{x \in \mathbb{R}^{n}}\right| x^{\alpha} D^{\beta} f(x) \mid<\infty, \forall \alpha, \beta \in \mathbb{Z}_{+}^{n}\right\},
$$

where $\alpha, \beta$ are multi-indexes, $\alpha=\left(\alpha_{1}, \cdots, \alpha_{n}\right), \beta=\left(\beta_{1}, \cdots, \beta_{n}\right)$,

$$
x^{\alpha}=x_{1}^{\alpha_{1}} x_{2}^{\alpha_{2}} \cdots x_{n}^{\alpha_{n}}, \quad D^{\beta} f=\frac{\partial^{\beta_{1}}}{\partial x_{1}^{\beta_{1}}} \cdots \frac{\partial^{\beta_{n}}}{\partial x_{n}^{\beta_{n}}} f .
$$


Let $\mathcal{S}^{\prime}$ be the space of all linear functionals on $\mathcal{S}$. Conventionally, $\mathcal{S}$ is called the Schwartz space, and $\mathcal{S}^{\prime}$ is called the space of Schwartz distributions or tempered distributions.

In order to define the Littlewood-Paley operators, we first introduce the following theorem on the partition of unity.

Theorem 2.1 ([17]). Suppose $\mathcal{C}$ is an annulus $\left\{\xi \in \mathbb{R}^{n}: 3 / 4 \leq|\xi| \leq 8 / 3\right\}$. Then there exist two radial functions $\hat{\chi}(\xi) \in \mathcal{D}\left(B_{\frac{4}{3}}(0)\right)$ and $\hat{\varphi}(\xi) \in \mathcal{D}(\mathcal{C})$ such that $0 \leq \hat{\chi}(\xi), \hat{\varphi}(\xi) \leq 1$ and

$$
\begin{array}{ll}
\hat{\chi}(\xi)+\sum_{j \geq 0} \hat{\varphi}\left(2^{-j} \xi\right)=1, & \forall \xi \in \mathbb{R}^{n}, \\
\sum_{j \in \mathbb{Z}} \hat{\varphi}\left(2^{-j} \xi\right)=1, & \forall \xi \in \mathbb{R}^{n} \backslash\{0\}, \\
\operatorname{supp} \hat{\varphi}\left(2^{-j} \cdot\right) \cap \operatorname{supp} \hat{\varphi}\left(2^{-j^{\prime}} \cdot\right)=\varnothing, & \left|j-j^{\prime}\right| \geq 2, \\
\operatorname{supp} \hat{\chi}(\cdot) \cap \operatorname{supp} \hat{\varphi}\left(2^{-j} \cdot\right)=\varnothing, & j \geq 1 .
\end{array}
$$

Based on the partition of unity on the spectrum space. We impose the following notations:

$$
\chi(x)=\mathcal{F}^{-1}(\hat{\chi}(\xi)), \quad \varphi(x)=\mathcal{F}^{-1}(\hat{\varphi}(\xi)),
$$

where $\mathcal{F}, \mathcal{F}^{-1}$ are Fourier and inverse Fourier transforms respectively, and define

$$
\left\{\begin{aligned}
\dot{\triangle}_{j} u:=\hat{\varphi}\left(2^{-j} D\right) u=2^{j n} \int_{\mathbb{R}^{n}} \varphi\left(2^{j} y\right) u(x-y) \mathrm{d} y, \quad j \in \mathbb{Z}, \\
\dot{S}_{j} u:=\hat{\chi}\left(2^{-j} D\right) u, \quad j \in \mathbb{Z} .
\end{aligned}\right.
$$

Definition 2.1 ([17]). Denote $\mathcal{S}_{h}^{\prime}$ the space of all Schwartz distributions $u$ satisfying

$$
\lim _{j \rightarrow-\infty} \dot{S}_{j} u=0, \quad u \in \mathcal{S}^{\prime}\left(\mathbb{R}^{n}\right) .
$$

Use the above notations, we obtain the Littlewood-Paley decomposition.

$$
u=\sum_{j \in \mathbb{Z}} \dot{\triangle}_{j} u, \quad u \in \mathcal{S}_{h}^{\prime}\left(\mathbb{R}^{n}\right) .
$$

Definition 2.2 (Homogeneous Besov space, [17]). Suppose $s \in \mathbb{R}, 1 \leq p, r \leq \infty$. Define

$$
\dot{B}_{p, r}^{s}=\left\{f \in \mathcal{S}_{h}^{\prime}\left(\mathbb{R}^{n}\right) \mid\|f\|_{\dot{B}_{p, r}^{s}}<\infty\right\},
$$

where

$$
\|f\|_{\dot{B}_{p, r}^{s}}=\left(\sum_{j \in \mathbb{Z}} 2^{r j s}\left\|\triangle_{j} f\right\|_{p}^{r}\right)^{1 / r}
$$

Proposition $2.1([17]) .\left(\dot{B}_{p, r}^{s},\|\cdot\|_{\dot{B}_{p, r}^{s}}\right)$ is a normed space. In particular, if $s<\frac{n}{p}$, then $\left(\dot{B}_{p, r}^{s}, \| \cdot\right.$ $\left.\|_{\dot{B}_{p, r}^{s}}\right)$ is a Banach space; For any $p \geq 1, \dot{B}_{p, 1}^{n / p}$ is also a Banach space. 
The space $\left(\dot{B}_{2,2^{\prime}}^{s},\|\cdot\|_{\dot{B}_{2,2^{\prime}}^{s}}\right)$ turns out to be the classical homogeneous Sobolev space $\dot{H}^{s}$. The following time-space Besov spaces are first introduced by Chemin and Lerner in Definition 2.1 of [4] for $r=p=2, a=1, s=n / 2+1$, and generalized later in [2].

Definition 2.3 (Definition 2.67 in [2]). Let $p, r, a \in[1, \infty]$ and $s \in \mathbb{R}$. Let $\tilde{L}_{T}^{a} \dot{B}_{p, r}^{s}=\tilde{L}_{T}^{a}\left(0, T ; \dot{B}_{p, r}^{s}\right)$ be the space of distributions $u \in \mathcal{S}_{h}^{\prime}$ such that

$$
\|u\|_{\tilde{L}_{T}^{a} \dot{B}_{p, r}^{s}}:=\left\|2^{j s}\right\| \dot{\triangle}_{j} u\left\|_{L^{a}\left(0, T ; L^{p}\right)}\right\|_{l^{r}(\mathbb{Z})}<\infty .
$$

For convenience, we use $\tilde{L}^{a} \dot{B}_{p, r}^{s}$ to represent the space with $T=+\infty$.

The spaces $\tilde{L}_{T}^{a} \dot{B}_{p, r}^{s}$ are related with the more classical ones $€[a] \dot{B}_{p, r}^{s}=€[a][]\left([0, T] ; \dot{B}_{p, r}^{s}\right)$ via the Minkowski inequality. Actually, one can prove that

$$
\|u\|_{\tilde{L}_{T}^{a} \dot{B}_{p, r}^{s}} \leq\|u\|_{€[a] \dot{B}_{p, r}^{s}} \quad \text { if } r \geq a, \quad\|u\|_{\tilde{L}_{T}^{a} \dot{B}_{p, r}^{s}} \geq\|u\|_{€[a] \dot{B}_{p, r}^{s}} \quad \text { if } r \leq a .
$$

All the properties of continuity for the product, composition, remainder, and paraproduct remain true in those spaces in general.

\subsection{Embeddings and inequalities}

Lemma 2.1 (Bernstein's inequalities, [11]). There exists a constant $C$ such that for any $q, k \in \mathbb{N}$, $1 \leq a \leq b$ and $f \in L^{a}\left(\mathbb{R}^{n}\right)$,

$$
\begin{aligned}
& \sup _{|\alpha|=k}\left\|\partial^{\alpha} \dot{S}_{q} f\right\|_{L^{b}} \leq C^{k} 2^{q\left(k+n\left(\frac{1}{a}-\frac{1}{b}\right)\right)}\left\|\dot{S}_{q} f\right\|_{L^{a}} \\
& C^{-k} 2^{q k}\left\|\dot{\triangle}_{q} f\right\|_{L^{a}} \leq \sup _{|\alpha|=k}\left\|\partial^{\alpha} \dot{\triangle}_{q} f\right\|_{L^{a}} \leq C^{k} 2^{q k}\left\|\dot{\triangle}_{q} f\right\|_{L^{a}} .
\end{aligned}
$$

Lemma 2.2 (Proposition 2.20 of [2]). Let $1 \leq p_{1} \leq p_{2} \leq \infty$ and $1 \leq r_{2} \leq r_{2} \leq \infty$. For any real number s, we have the continuous embedding

$$
\dot{B}_{p_{1}, r_{1}}^{s}\left(\mathbb{R}^{n}\right) \hookrightarrow \dot{B}_{p_{2}, r_{2}}^{s-n\left(1 / p_{1}-1 / p_{2}\right)}\left(\mathbb{R}^{n}\right) .
$$

Lemma 2.3 (Proposition 2.39 of [2]). For $1 \leq p \leq q \leq \infty$, we have the continuous embedding

$$
\dot{B}_{p, 1}^{n / p-n / q}\left(\mathbb{R}^{n}\right) \hookrightarrow L^{q}\left(\mathbb{R}^{n}\right) .
$$

Lemma 2.4 ([2]). We have the time estimate

$$
\|u v\|_{\tilde{L}_{T}^{a} \dot{B}_{p, r}^{s}} \leq C\left(\|u\|_{L_{T}^{a_{1}}\left(L^{\infty}\right)}\|v\|_{\tilde{L}_{T}^{a_{2}} \dot{B}_{p, r}^{s}}+\|v\|_{\tilde{L}_{T}^{a_{3}}\left(L^{\infty}\right)}\|u\|_{\tilde{L}_{T}^{a_{3}} \dot{B}_{p, r}^{s}}\right),
$$

whenever $s>0, p, a, a_{1}, a_{2}, a_{3}, a_{4} \in[1, \infty]$ and

$$
\frac{1}{a}=\frac{1}{a_{1}}+\frac{1}{a_{2}}=\frac{1}{a_{3}}+\frac{1}{a_{4}} .
$$


Let $u$ and $v$ be tempered distributions in $\mathcal{S}_{h}^{\prime}\left(\mathbb{R}^{n}\right)$. We have by (2.2) that

$$
u v=\sum_{j, j^{\prime} \in \mathbb{Z}} \dot{\triangle}_{j^{\prime}} u \dot{\triangle}_{j} v \text {. }
$$

The key technique of paradifferential calculus is the following Bony decomposition

$$
u v=\dot{T}_{u} v+\dot{T}_{v} u+\dot{R}(u, v), \quad u, v \in \mathcal{S}_{h}^{\prime}\left(\mathbb{R}^{n}\right),
$$

where

$$
\dot{T}_{u} v:=\sum_{j \in \mathbb{Z}} \dot{S}_{j-1} u \dot{\triangle}_{j} v, \quad \dot{R}(u, v):=\sum_{\left|j-j^{\prime}\right| \leq 1} \dot{\triangle}_{j^{\prime}} u \dot{\triangle}_{j} v .
$$

Lemma 2.5 (Theorem 2.47 of [2]). Let $s \in \mathbb{R}$ and $t<0$. There exists a constant $C=C(s, t)$ such that for any $p, r_{1}, r_{2} \in[1, \infty], u \in \dot{B}_{\infty, r_{1}}^{t}$ and $v \in \dot{B}_{p, r_{2}}^{s}$,

$$
\left\|\dot{T}_{u} v\right\|_{\dot{B}_{p, r}^{s, t}} \leq C\|u\|_{\dot{B}_{\infty, r_{1}}^{t}}\|v\|_{\dot{B}_{p, r_{2}}^{s}}
$$

with $\frac{1}{r}=\min \left\{1, \frac{1}{r_{1}}+\frac{1}{r_{2}}\right\}$.

Lemma 2.6 (Theorem 2.52 of [2]). Let $s_{1}, s_{2} \in \mathbb{R}$ such that $s_{1}+s_{2}>0$. There exists a constant $C=C\left(s_{1}, s_{2}\right)$ such that, for any $p_{1}, p_{2}, r_{1}, r_{2} \in[1, \infty], u \in \dot{B}_{p_{1}, r_{1}}^{s_{1}}$ and $v \in \dot{B}_{p_{2}, r_{2}}^{s_{2}}$,

$$
\|\dot{R}(u, v)\|_{\dot{B}_{p, r}^{s_{1}+s_{2}}} \leq C\|u\|_{\dot{B}_{p_{1}, r_{1}}^{s_{1}}}\|v\|_{\dot{B}_{p_{2}, r_{2}}^{s_{2}}},
$$

provided that $\frac{1}{p}:=\frac{1}{p_{1}}+\frac{1}{p_{2}} \leq 1$ and $\frac{1}{r}:=\frac{1}{r_{1}}+\frac{1}{r_{2}} \leq 1$.

\section{A priori estimates}

In this section, we consider some properties of the Stokes and Maxwell equations.

Multiplying the first line of (1.1) by $u$, the second line by $E$, the third line by $B$, adding them together, then integrating over $\mathbb{R}^{n}$, we obtain the well-known energy equality

$$
\frac{d}{d t}\left(\|u\|_{L^{2}}^{2}+\|E\|_{L^{2}}^{2}+\|B\|_{L^{2}}^{2}\right)+\|\nabla u\|_{L^{2}}^{2}+\sigma^{-1}\|j\|_{L^{2}}^{2}=0 .
$$

From the regularity theory of parabolic type system, we have the following estimate in Besov spaces for the Stokes equation.

Proposition 3.1 (Theorem 3.37 \& Remark 3.39 in [2], Lemma 2.3 in [11]). Let $u$ be a smooth divergence free vector field solving the Stokes equation

$$
\begin{cases}\partial_{t} u-\triangle u+\nabla p=f, & (t, x) \in[0, T] \times \mathbb{R}^{3}, \\ \left.u\right|_{t=0}=u_{0}, & x \in \mathbb{R}^{3} .\end{cases}
$$

Then for every $1 \leq r \leq p$ and $s \in \mathbb{R}$, there exists a constant $C$, independent of $T$, such that

$$
\|u\|_{C\left([0, T] ; \dot{B}_{q, 1}^{s}\right) \cap \tilde{L}_{T}^{p} \dot{\dot{B}}_{q, 1}^{s+2 / p}} \leq C\left(\left\|u_{0}\right\|_{\dot{B}_{q, 1}^{s}}+\|f\|_{\tilde{L}_{T}^{r} B_{q, 1}^{s-2+2 / r}}\right) .
$$


We can apply energy estimate on the Maxwell system in our desired Besov spaces.

Proposition 3.2. If $(E, B)$ solves equations

$$
\left\{\begin{array}{l}
E_{t}-\nabla \times B+E=f, \\
B_{t}+\nabla \times E=0, \\
\operatorname{div} B=0, \\
B(x, 0)=B_{0}(x), \quad E(x, 0)=E_{0}(x),
\end{array}\right.
$$

on $[0, T]$, then there is a constant $C$, which is independent of $T$, such that

$$
\|E(t)\|_{\tilde{L}_{T}^{\infty} \dot{B}_{2,1}^{1 / 2} \cap \tilde{L}_{T}^{2} \dot{B}_{2,1}^{1 / 2}}+\|B(t)\|_{\tilde{L}_{T}^{\infty} \dot{B}_{2,1}^{1 / 2}} \leq C\left(\left\|E_{0}\right\|_{\dot{B}_{2,1}^{1 / 2}}+\left\|B_{0}\right\|_{\dot{B}_{2,1}^{1 / 2}}+\|f\|_{\tilde{L}_{T}^{2} \dot{B}_{2,1}^{1 / 2}}\right) .
$$

Proof. Applying the homogeneous Littlewood-Paley operator $\dot{\triangle}_{j}$ to the first and second line of (3.1), we obtain

$$
\begin{aligned}
& \frac{\partial}{\partial t} \dot{\triangle}_{j} E-\dot{\triangle}_{j}(\nabla \times B)+\dot{\triangle}_{j} E=\dot{\triangle}_{j} f, \\
& \frac{\partial}{\partial t} \dot{\triangle}_{j} B+\dot{\triangle}_{j}(\nabla \times E)=0 .
\end{aligned}
$$

Adding the inner product of (3.3) and $\triangle_{j} E$ to the inner product of (3.4) and $\triangle_{j} B$, then integrating with respect to $x$ over $\mathbb{R}^{3}$, we have that

$$
\frac{1}{2} \frac{\mathrm{d}}{\mathrm{d} t}\left\|\dot{\triangle}_{j} E\right\|_{L^{2}}^{2}+\frac{1}{2} \frac{\mathrm{d}}{\mathrm{d} t}\left\|\dot{\triangle}_{j} B\right\|_{L^{2}}^{2}+\left\|\dot{\triangle}_{j} E\right\|_{L^{2}}^{2}=\dot{\triangle}_{j} E \cdot \dot{\triangle}_{j} f \leq \varepsilon\left\|\dot{\triangle}_{j} E\right\|_{L^{2}}^{2}+\frac{C}{\varepsilon}\left\|\dot{\triangle}_{j} f\right\|_{L^{2}}^{2},
$$

using integration by parts and Young's inequality.

Let $M(t):=\left\|\dot{\triangle}_{j} E\right\|_{L^{2}}^{2}+\left\|\dot{\triangle}_{j} B\right\|_{L^{2}}^{2}$ and fix $\varepsilon=1 / 2$, so $M_{t} \leq C\left\|\triangle_{j} f\right\|_{L^{2}}^{2}$.

$$
\left\|\triangle_{j} B\right\|_{L^{2}}^{2} \leq M \leq M(0)+C\left\|\triangle_{j} f\right\|_{L_{t}^{2} L^{2}}^{2}
$$

The above inequality and (3.5) yield

$$
\frac{\mathrm{d}}{\mathrm{d} t} M+M \leq C M(0)+C\left\|\dot{\triangle}_{j} f\right\|_{L^{2}}^{2}+C\left\|\dot{\triangle}_{j} f\right\|_{L_{t}^{2} L^{2}}^{2}
$$

By Grönwall-type inequality, we know that

$$
\begin{aligned}
& M(t) \leq e^{-t} M(0)+\int_{0}^{t} e^{(s-t)}\left(C M(0)+C\left\|\dot{\triangle}_{j} f\right\|_{L^{2}}^{2}(s)+C\left\|\dot{\triangle}_{j} f\right\|_{L_{s}^{2} L^{2}}^{2}\right) \mathrm{d} s \\
\leq & C M(0)+C\left\|\dot{\triangle}_{j} f\right\|_{L_{t}^{2} L^{2}}^{2} .
\end{aligned}
$$

Taking supremum over $[0, T]$ of (3.6), we obtain that

$$
\left\|\dot{\triangle}_{j} E\right\|_{L_{T}^{\infty} L^{2}}^{2}+\left\|\dot{\triangle}_{j} B\right\|_{L_{T}^{\infty} L^{2}}^{2} \leq C\left\|\dot{\triangle}_{j} E_{0}\right\|_{L^{2}}^{2}+C\left\|\dot{\triangle}_{j} B_{0}\right\|_{L^{2}}^{2}+C\left\|\dot{\triangle}_{j} f\right\|_{L_{T}^{2} L^{2}}^{2} .
$$


Therefore

$$
\|E\|_{\tilde{L}_{T}^{\infty} \dot{B}_{2,1}^{1 / 2}}+\|B\|_{\tilde{L}_{T}^{\infty} \dot{B}_{2,1}^{1 / 2}} \leq C\left(\left\|E_{0}\right\|_{\dot{B}_{2,1}^{1 / 2}}+\left\|B_{0}\right\|_{\dot{B}_{2,1}^{1 / 2}}+\|f\|_{\tilde{L}_{T}^{2} \dot{B}_{2,1}^{1 / 2}}\right) .
$$

Taking integral of (3.6) over $[0, T]$, we obtain by Fubini's lemma that

$$
\begin{aligned}
& \left\|\dot{\triangle}_{j} E\right\|_{L_{T}^{2} L^{2}}^{2}+\left\|\dot{\triangle}_{j} B\right\|_{L_{T}^{2} L^{2}}^{2} \\
\leq & C\left(\int_{0}^{T} e^{-t} \mathrm{~d} t+\int_{0}^{T} \int_{0}^{t} e^{s-t} \mathrm{~d} s \mathrm{~d} t\right) M(0)+C \int_{0}^{T} \int_{0}^{t} e^{(s-t)}\left(\left\|\dot{\triangle}_{j} f\right\|_{L^{2}}^{2}(s)+\left\|\dot{\triangle}_{j} f\right\|_{L_{s}^{2} L^{2}}^{2}\right) \mathrm{d} s \mathrm{~d} t \\
\leq & C M(0)+C\left\|\dot{\triangle}_{j} f\right\|_{L_{T}^{2} L^{2}}^{2}
\end{aligned}
$$

where the constant $C$ is independent of $T$. Estimate (3.2) is true.

\section{Proof of the Main Theorem}

\subsection{One useful lemma}

Lemma 4.1. There exists a constant $C$ such that

$$
\begin{aligned}
& \|f g\|_{\tilde{L}_{T}^{2} \dot{B}_{2,1}^{-1 / 2}} \leq C\|f\|_{\tilde{L}_{T}^{2} \dot{B}_{2,1}^{1 / 2}}\|g\|_{\tilde{L}_{T}^{\sigma_{1}} \dot{B}_{2,1}^{1 / 2}}, \\
& \|f g\|_{L_{T}^{2} \dot{B}_{2,1}^{1 / 2}} \leq C\|f\|_{\tilde{L}_{T}^{2} \dot{B}_{2,1}^{3 / 2}}\|g\|_{\tilde{L}_{T}^{\sigma_{B}^{\infty}} \dot{B}_{2,1}^{1 / 2},} \\
& \|g \nabla f\|_{\tilde{L}_{T}^{2} \dot{B}_{2,1}^{-1 / 2}} \leq C\|f\|_{\tilde{L}_{T}^{2} \dot{B}_{2,1}^{3 / 2}} \cdot\|g\|_{\tilde{L}_{T}^{\infty} \dot{B}_{2,1}^{1 / 2}}
\end{aligned}
$$

for all smooth functions $f, g$ defined on some interval $[0, T]$.

Proof. The inequality (4.3) can be obtained by replacing $f$ by $\nabla f$ in (4.1). So we only need to prove (4.1) and (4.2).

Step 1. To prove (4.1). By Bony's decomposition, $f g=\dot{T}_{f} g+\dot{T}_{g} f+\dot{R}(f, g)$. And we have by Bernstein's inequality that

$$
\begin{aligned}
\left\|\dot{T}_{g} f\right\|_{\tilde{L}_{T}^{2} \dot{B}_{2,1}^{-1 / 2}} & \leq C \sum_{j} 2^{-j / 2}\left\|\dot{\triangle}_{j} \dot{T}_{g} f\right\|_{L_{T}^{2} L^{2}} \\
& \leq C \sum_{j} 2^{-j / 2}\left\|\dot{\triangle}_{j} f\right\|_{L_{T}^{2} L^{2}}\left\|S_{j-1} g\right\|_{L_{T}^{\infty} L^{\infty}} \\
& \leq C \sum_{j} 2^{-j / 2}\left\|\dot{\triangle}_{j} f\right\|_{L_{T}^{2} L^{2}}\left(\sum_{k \leq j-2} 2^{3 k / 2}\left\|\dot{\triangle}_{k} g\right\|_{L_{T}^{\infty} L^{2}}\right) \\
& \leq C \sum_{j} 2^{j / 2}\left\|\dot{\triangle}_{j} f\right\|_{L_{T}^{2} L^{2}}\left(\sum_{k \leq j-2} 2^{k-j} 2^{k / 2}\left\|\dot{\triangle}_{k} g\right\|_{L_{T}^{\infty} L^{2}}\right) \\
& \leq C\left(\sum_{j} 2^{j / 2}\left\|\triangle_{j} f\right\|_{L_{T}^{2} L^{2}}\right)\left(\sum_{k} 2^{k / 2}\left\|\dot{\triangle}_{k} g\right\|_{L_{T}^{\infty} L^{2}}\right) \leq C\|f\|_{\tilde{L}_{T}^{2} \dot{B}_{2,1}^{1 / 2}}\|g\|_{\tilde{L}_{T}^{\infty} \dot{B}_{2,1}^{1 / 2}} .
\end{aligned}
$$


Similarly,

$$
\begin{aligned}
\left\|\dot{T}_{f} g\right\|_{\tilde{L}_{T}^{2} \dot{B}_{2,1}^{-1 / 2}} & \leq C \sum_{j} 2^{-j / 2}\left\|\dot{\triangle}_{j} \dot{T}_{f} g\right\|_{L_{T}^{2} L^{2}} \\
& \leq C \sum_{j} 2^{-j / 2}\left\|\dot{\triangle}_{j} g\right\|_{L_{T}^{\infty} L^{2}}\left\|S_{j-1} f\right\|_{L_{T}^{2} L^{\infty}} \\
& \leq C \sum_{j} 2^{j / 2}\left\|\dot{\triangle}_{j} g\right\|_{L_{T}^{\infty} L^{2}}\left(\sum_{k \leq j-2} 2^{k-j} 2^{k / 2}\left\|\dot{\triangle}_{k} f\right\|_{L_{T}^{2} L^{2}}\right) \leq C\|f\|_{\tilde{L}_{T}^{2} \dot{B}_{2,1}^{s} 1 / 2}\|g\|_{\tilde{L}_{T}^{\infty} \dot{B}_{2,1}^{1 / 2}} .
\end{aligned}
$$

The remaining term

$$
\begin{aligned}
\|R(f, g)\|_{\tilde{L}_{T}^{2} \dot{B}_{2,1}^{-1 / 2}} & \leq C \sum_{j} 2^{-j / 2}\left\|\dot{\triangle}_{j} R(f, g)\right\|_{L_{T}^{2} L^{2}} \\
& \leq C \sum_{\left|j-j^{\prime}\right| \leq 1} 2^{-j / 2}\left\|\dot{\triangle}_{j} f\right\|_{L_{T}^{2} L^{2}}\left\|\dot{\triangle}_{j^{\prime}} g\right\|_{L_{T}^{\infty} L^{\infty}} \\
& \leq C \sum_{\left|j-j^{\prime}\right| \leq 1} 2^{-j / 2}\left\|\dot{\triangle}_{j} f\right\|_{L_{T}^{2} L^{2}} 2^{3 j^{\prime} / 2}\left\|\dot{\triangle}_{j^{\prime}} g\right\|_{L_{T}^{\infty} L^{2}} \leq C\|f\|_{\tilde{L}_{T}^{2} \dot{B}_{2,1}^{1 / 2}}\|g\|_{\tilde{L}_{T}^{\infty} \dot{B}_{2,1}^{1 / 2}} .
\end{aligned}
$$

Step 2. To prove (4.2). By Bony's decomposition and Bernstein's inequality similarly, we have

$$
\begin{aligned}
\left\|\dot{T}_{g} f\right\|_{\tilde{L}_{T}^{2} \dot{B}_{2,1}^{1 / 2}} & \leq C \sum_{j} 2^{j / 2}\left\|\dot{\triangle}_{j} \dot{T}_{g} f\right\|_{L_{T}^{2} L^{2}} \\
& \leq C \sum_{j} 2^{j / 2}\left\|\dot{\triangle}_{j} f\right\|_{L_{T}^{2} L^{2}}\left\|S_{j-1} g\right\|_{L_{T}^{\infty} L^{\infty}} \\
& \leq C \sum_{j} 2^{j / 2}\left\|\dot{\triangle}_{j} f\right\|_{L_{T}^{2} L^{2}}\left(\sum_{k \leq j-2} 2^{3 k / 2}\left\|\dot{\triangle}_{k} g\right\|_{L_{T}^{\infty} L^{2}}\right) \\
& \leq C \sum_{j} 2^{3 j / 2}\left\|\dot{\triangle}_{j} f\right\|_{L_{T}^{2} L^{2}}\left(\sum_{k \leq j-2} 2^{k-j} 2^{k / 2}\left\|\dot{\triangle}_{k} g\right\|_{L_{T}^{\infty} L^{2}}\right) \\
& \leq C\left(\sum_{j} 2^{3 j / 2}\left\|\dot{\triangle}_{j} f\right\|_{L_{T}^{2} L^{2}}\right)\left(\sum_{k} 2^{k / 2}\left\|\dot{\triangle}_{k} g\right\|_{L_{T}^{\infty} L^{2}}\right) \leq C\|f\|_{\tilde{L}_{T}^{2} \dot{B}_{2,1}^{3 / 2}}\|g\|_{\tilde{L}_{T}^{\infty} \dot{B}_{2,1}^{1 / 2}} .
\end{aligned}
$$

Similar inequlities of $\dot{T}_{f} g$ and $\dot{R}(f, g)$ can be derived similarl. Hence the inequality (4.2) holds.

Now we are ready to prove the main result of this paper.

\subsection{Proof of Theorem 1.1 .}

Step 1. Existence 
Set $u_{0}=E_{0}=B_{0}=0$. We use the following iteration scheme to construct a sequence of approximate solutions $\left(u_{n}, E_{n}, B_{n}\right)$ on $\mathbb{R}^{+} \times \mathbb{R}^{3}$.

$$
\left\{\begin{array}{l}
\partial_{t} u_{n+1}-\triangle u_{n+1}+\nabla p_{n+1}=-u_{n} \cdot \nabla u_{n}+E_{n} \times B_{n}+\left(u_{n} \times B_{n}\right) \times B_{n}, \\
\partial_{t} E_{n+1}-\operatorname{curl} B_{n+1}+E_{n+1}=-u_{n} \times B_{n}, \\
\partial_{t} B_{n+1}+\operatorname{curl} E_{n+1}=0, \\
\operatorname{div} B_{n+1}=\operatorname{div} u_{n+1}=0, \\
\left.u_{n+1}\right|_{t=0}=S_{\theta_{n+1}} u_{0},\left.\quad E_{n+1}\right|_{t=0}=S_{\theta_{n+1}} E_{0},\left.\quad B_{n+1}\right|_{t=0}=S_{\theta_{n+1}} B_{0} .
\end{array}\right.
$$

Since $\lim _{j \rightarrow+\infty} S_{j}=I$, the sequence $\theta_{n+1} \in \mathbb{N}$ can be chosen such that

$$
\left\|\left(S_{\theta_{n+1}}-S_{\theta_{n}}\right)\left(u_{0}, E_{0}, B_{0}\right)\right\|_{\mathbf{X}} \leq 2^{-n} .
$$

Denote

$$
\mathbf{Z}=\left(\tilde{L}^{\infty} \dot{B}_{2,1}^{1 / 2} \cap \tilde{L}^{2} \dot{B}_{2,1}^{3 / 2}\right) \times\left(\tilde{L}^{\infty} \dot{B}_{2,1}^{1 / 2} \cap \tilde{L}^{2} \dot{B}_{2,1}^{1 / 2}\right) \times \tilde{L}^{\infty} \dot{B}_{2,1}^{1 / 2}
$$

Obviously, we have by Lemma 4.1 that

$$
\begin{aligned}
& \left\|u_{n} \cdot \nabla u_{n}+E_{n} \times B_{n}+\left(u_{n} \times B_{n}\right) \times B_{n}\right\|_{\tilde{L}^{2} \dot{B}_{2,1}^{-1 / 2}} \\
\leq & C\left(\left\|u_{n}\right\|_{\tilde{L}^{2} \dot{B}_{2,1}^{3 / 2}}\left\|u_{n}\right\|_{\tilde{L}^{\infty} \dot{B}_{2,1}^{1 / 2}}+\|E\|_{\tilde{L}^{2} \dot{B}_{2,1}^{1 / 2}}\|B\|_{\tilde{L}^{\infty} \dot{B}_{2,1}^{1 / 2}}+\|u\|_{\tilde{L}^{2} \dot{B}_{2,1}^{3 / 2}}\|B\|_{\tilde{L}^{\infty} \dot{B}_{2,1}^{1 / 2}}^{2}\right) .
\end{aligned}
$$

By Proposition 3.1, Proposition 3.2 and Lemma 4.1, the family of solutions $\left(u_{n}, E_{n}, B_{n}\right)$ are well-defined and

$$
\begin{aligned}
& \left\|u_{n+1}\right\|_{\tilde{L}^{\infty} \dot{B}_{2,1}^{1 / 2} \cap \tilde{L}^{2} \dot{B}_{2,1}^{3 / 2}} \\
& \quad \leq C\left(\left\|u_{n+1}(0)\right\|_{\dot{B}_{2,1}^{1 / 2}}+\left\|u_{n}\right\|_{\tilde{L}^{2} \dot{B}_{2,1}^{3 / 2}}\left\|u_{n}\right\|_{\tilde{L}^{\infty} \dot{B}_{2,1}^{1 / 2}}+\|E\|_{\tilde{L}^{2} \dot{B}_{2,1}^{1 / 2}}\|B\|_{\tilde{L}^{\infty} \dot{B}_{2,1}^{1 / 2}}+\|u\|_{\tilde{L}^{2} \dot{B}_{2,1}^{3 / 2}}\|B\|_{\tilde{L}^{\infty} \dot{B}_{2,1}^{1 / 2}}^{2}\right) \\
& \quad\left\|E_{n+1}\right\|_{\tilde{L}^{\infty} \dot{B}_{2,1}^{1 / 2} \cap \tilde{L}^{2} \dot{B}_{2,1}^{1 / 2}}+\left\|B_{n+1}\right\|_{\tilde{L}^{\infty} \dot{B}_{2,1}^{1 / 2}} \\
& \quad \leq C\left(\left\|E_{n+1}(0)\right\|_{\dot{B}_{2,1}^{1 / 2}}+\left\|B_{n+1}(0)\right\|_{\dot{B}_{2,1}^{1 / 2}}+\left\|u_{n}\right\|_{\tilde{L}^{2} \dot{B}_{2,1}^{3 / 2}}\left\|B_{n}\right\|_{\tilde{L}^{\infty} \dot{B}_{2,1}^{1 / 2}}\right) .
\end{aligned}
$$

Let $\mathcal{E}_{n}:=\left\|\left(u_{n}, E_{n}, B_{n}\right)\right\|_{\mathbf{z}}=\left\|u_{n}\right\|_{\tilde{L}^{\infty} \dot{B}_{2,1}^{1 / 2} \cap \tilde{L}^{2} \dot{B}_{2,1}^{3 / 2}}+\left\|E_{n}\right\|_{\tilde{L}^{\infty} \dot{B}_{2,1}^{1 / 2} \cap \tilde{L}^{2} \dot{B}_{2,1}^{1 / 2}}+\left\|B_{n}\right\|_{\tilde{L}^{\infty} \dot{B}_{2,1}^{1 / 2}}$. Then the last two estimates imply that

$$
\mathcal{E}_{n+1} \leq C\left(\left\|u_{n+1}(0)\right\|_{\dot{B}_{2,1}^{1 / 2}}+\left\|E_{n+1}(0)\right\|_{\dot{B}_{2,1}^{1 / 2}}+\left\|B_{n+1}(0)\right\|_{\dot{B}_{2,1}^{1 / 2}}\right)+C \mathcal{E}_{n}^{2}+C \mathcal{E}_{n}^{3} .
$$

Note that

$$
\begin{aligned}
& \left\|u_{n+1}(0)\right\|_{\dot{B}_{2,1}^{1 / 2}}+\left\|E_{n+1}(0)\right\|_{\dot{B}_{2,1}^{1 / 2}}+\left\|B_{n+1}(0)\right\|_{\dot{B}_{2,1}^{1 / 2}} \\
= & \left\|S_{\theta_{n+1}} u_{0}\right\|_{\dot{B}_{2,1}^{1 / 2}}+\left\|S_{\theta_{n+1}} E_{0}\right\|_{\dot{B}_{2,1}^{1 / 2}}+\left\|S_{\theta_{n+1}} B_{0}\right\|_{\dot{B}_{2,1}^{1 / 2}} \\
\leq & \left\|u_{0}\right\|_{\dot{B}_{2,1}^{1 / 2}}+\left\|E_{0}\right\|_{\dot{B}_{2,1}^{1 / 2}}+\left\|B_{0}\right\|_{\dot{B}_{2,1}^{1 / 2}} \leq \delta .
\end{aligned}
$$


So by (4.4) and $\mathcal{E}_{0}=0$, we have that $\mathcal{E}_{n} \leq C \delta$ for any $n \in \mathbb{N}$ for $\delta$ sufficiently small. Set for $n \geq 0$ that

$$
\begin{aligned}
\mathcal{D}_{n} & :=\left\|\left(u_{n+1}-u_{n}, E_{n+1}-E_{n}, B_{n+1}-B_{n}\right)\right\|_{\mathbf{z}} \\
& =\left\|u_{n+1}-u_{n}\right\|_{\tilde{L}^{\infty} \dot{B}_{2,1}^{1 / 2} \cap \tilde{L}^{2} \dot{B}_{2,1}^{3 / 2}}+\left\|E_{n+1}-E_{n}\right\|_{\tilde{L}^{\infty} \dot{B}_{2,1}^{1 / 2} \cap \tilde{L}^{2} \dot{B}_{2,1}^{1 / 2}}+\left\|B_{n+1}-B_{n}\right\|_{\tilde{L}^{\infty} \dot{B}_{2,1}^{1 / 2}} .
\end{aligned}
$$

Similar arguments applied to $u_{n+1}-u_{n}$ imply that

$$
\begin{aligned}
\mathcal{D}_{n} & \leq C\left\|\left(S_{\theta_{n+1}}-S_{\theta_{n}}\right)\left(u_{0}, E_{0}, B_{0}\right)\right\|_{\mathbf{x}}+C \mathcal{D}_{n-1}\left(\mathcal{E}_{n-1}+\mathcal{E}_{n-1}^{2}+\mathcal{E}_{n}+\mathcal{E}_{n}^{2}\right) \\
& \leq C 2^{-n}+C \delta \mathcal{D}_{n-1} .
\end{aligned}
$$

Hence $\mathcal{D}_{n}$ is a Cauchy sequence in $\mathbb{R}$ and $\left(u_{n}, E_{n}, B_{n}\right)$ is a Cauchy sequence in $\mathbf{Z}$, so it has a limit $(u, E, B)$ which is a mild solution of (1.1).

Step 2. Uniqueness

It is sufficiently to prove that every solution of $(1.1)$ with initial data $\left(u_{0}, E_{0}, B_{0}\right)$ coincides with the small solution we constructed in Step 1. Denote

$$
\mathbf{Z}_{T}=\left(\tilde{L}_{T}^{\infty} \dot{B}_{2,1}^{1 / 2} \cap \tilde{L}_{T}^{2} \dot{B}_{2,1}^{3 / 2}\right) \times\left(\tilde{L}_{T}^{\infty} \dot{B}_{2,1}^{1 / 2} \cap \tilde{L}_{T}^{2} \dot{B}_{2,1}^{1 / 2}\right) \times \tilde{L}_{T}^{\infty} \dot{B}_{2,1}^{1 / 2} .
$$

Let $\left(u_{1}, E_{1}, B_{1}\right)$ be the small solution in $\mathbf{Z}$ we constructed in Step 1 , and $\left(u_{2}, E_{2}, B_{2}\right)$ be another solution in $\mathbf{Z}_{T}$ and has the same initial data. The difference $(\tilde{u}, \tilde{E}, \tilde{B})=\left(u_{1}, E_{1}, B_{1}\right)-$ $\left(u_{2}, E_{2}, B_{2}\right)$ solves the system

$$
\left\{\begin{array}{l}
\tilde{u}_{t}-\triangle \tilde{u}+\nabla \tilde{p}=G \\
\tilde{E}_{t}-\nabla \times \tilde{B}+\tilde{E}=H, \\
\tilde{B}_{t}+\nabla \times \tilde{E}=0, \\
\operatorname{div} \tilde{u}=0, \quad \operatorname{div} \tilde{B}=0, \\
\tilde{u}(x, 0)=\tilde{B}(x, 0)=\tilde{E}(x, 0)=0,
\end{array}\right.
$$

where

$$
\begin{aligned}
& G=\tilde{E} \times B_{1}+E_{2} \times \tilde{B}+\tilde{u} \times B_{1} \times B_{1}+u_{2} \times\left(\tilde{B} \times B_{1}+B_{2} \times \tilde{B}\right)-\tilde{u} \cdot \nabla u_{2}-u_{1} \cdot \nabla \tilde{u}, \\
& H=-\tilde{u} \times B_{1}-u_{2} \times \tilde{B} .
\end{aligned}
$$

By Proposition 3.1, Proposition 3.2 and Lemma 4.1, we know that

$$
\begin{aligned}
& \|(\tilde{u}, \tilde{E}, \tilde{B})\|_{\mathbf{z}_{T}} \leq C\|G\|_{\tilde{L}_{T}^{2} \dot{B}_{2,1}^{-1 / 2}}+C\|H\|_{\tilde{L}_{T}^{2} \dot{B}_{2,1}^{1 / 2}} \\
\leq & C\left(\|\tilde{E}\|_{\tilde{L}_{T}^{2} \dot{B}_{2,1}^{1 / 2}}\left\|B_{1}\right\|_{\tilde{L}_{T}^{\infty} \dot{B}_{2,1}^{1 / 2}}+\|\tilde{B}\|_{\tilde{L}_{T}^{\infty} \dot{B}_{2,1}^{1 / 1}}\left\|E_{2}\right\|_{\tilde{L}_{T}^{2} \dot{B}_{2,1}^{1 / 2}}+\|\tilde{u}\|_{\tilde{L}_{T}^{2} \dot{B}_{2,1}^{3 / 2}}\left\|B_{1}\right\|_{\tilde{L}_{T}^{\infty} \dot{B}_{2,1}^{1 / 2}}^{2}\right. \\
& +\|\tilde{B}\|_{\tilde{L}_{T}^{\infty} \dot{B}_{2,1}^{1 / 2}}\left\|u_{2}\right\|_{\tilde{L}_{T}^{2} \dot{B}_{2,1}^{3 / 2}}\left(\left\|B_{1}\right\|_{\tilde{L}_{T}^{\infty} \dot{B}_{2,1}^{1 / 2}}+\left\|B_{2}\right\|_{\tilde{L}_{T}^{\infty} \dot{B}_{2,1}^{1 / 2}}\right) \\
& \left.+\|\tilde{u}\|_{\tilde{L}_{T}^{\infty} \dot{B}_{2,1}^{1 / 2}} \cdot\left\|u_{2}\right\|_{\tilde{L}_{T}^{2} \dot{B}_{2,1}^{3 / 2}}+\|\tilde{u}\|_{\tilde{L}_{T}^{2} \dot{B}_{2,1}^{3 / 2}} \cdot\left\|u_{1}\right\|_{\tilde{L}_{T}^{\infty} \dot{B}_{2,1}^{1 / 2}}\right)
\end{aligned}
$$




$$
\begin{aligned}
\leq & C\|(\tilde{u}, \tilde{E}, \tilde{B})\|_{\mathbf{z}_{T}}\left(\left\|B_{1}\right\|_{\tilde{L}_{T}^{\infty} \dot{B}_{2,1}^{1 / 2}}+\left\|B_{1}\right\|_{\tilde{L}_{T}^{\infty} \dot{B}_{2,1}^{1 / 2}}^{2}+\left\|u_{1}\right\|_{\tilde{L}_{T}^{\infty} \dot{B}_{2,1}^{1 / 2}}\right. \\
& \left.+\left\|E_{2}\right\|_{\tilde{L}_{T}^{2} \dot{B}_{2,1}^{1 / 2}}+\left\|u_{2}\right\|_{\tilde{L}_{T}^{2} \dot{B}_{2,1}^{3 / 2}}\left(1+\left\|B_{1}\right\|_{\tilde{L}_{T}^{\infty} \dot{B}_{2,1}^{1 / 2}}+\left\|B_{2}\right\|_{\tilde{L}_{T}^{\infty} \dot{B}_{2,1}^{1 / 2}}\right)\right) .
\end{aligned}
$$

Noting that the norm of $u_{1}, B_{1}$ are small due to its construction, and $\left\|u_{2}\right\|_{\tilde{L}_{T}^{2} \dot{B}_{2,1}^{3 / 2}}\left\|E_{2}\right\|_{\tilde{L}_{T}^{2} \dot{B}_{2,1}^{1 / 2}}$ are small for $T$ sufficiently small. We can choose $T<<1$ such that

$$
\begin{aligned}
& C\left(\left\|B_{1}\right\|_{\tilde{L}_{T}^{\infty} \dot{B}_{2,1}^{1 / 2}}+\left\|B_{1}\right\|_{\tilde{L}_{T}^{\infty} \dot{B}_{2,1}^{1 / 2}}^{2}+\left\|u_{1}\right\|_{\tilde{L}_{T}^{\infty} \dot{B}_{2,1}^{1 / 2}}\right. \\
& \left.+\left\|E_{2}\right\|_{\tilde{L}_{T}^{2} \dot{B}_{2,1}^{1 / 2}}+\left\|u_{2}\right\|_{\tilde{L}_{T}^{2} \dot{B}_{2,1}^{3 / 2}}\left(1+\left\|B_{1}\right\|_{\tilde{L}_{T}^{\infty} \dot{B}_{2,1}^{1 / 2}}+\left\|B_{2}\right\|_{\tilde{L}_{T}^{\infty} \dot{B}_{2,1}^{1 / 2}}\right)\right)<1 .
\end{aligned}
$$

So $\|(\tilde{u}, \tilde{E}, \tilde{B})\|_{\mathbf{z}_{T}}=0$, the solution is unique on $[0, T]$. Iterating on intervals $[k T,(k+1) T]$, $k=1,2, \cdots$, yields the uniqueness on $[0, \infty)$.

Remark 4.1. Similar to [11], we proved the global existence for small initial data. Noting that the $L_{T}^{\infty}$ norm does not necessarily decrease as $T$ goes to zero, so $\|(u, E, B)\|_{\mathbf{z}_{T}}$ is not small for $0<T<<1$. Therefore, local-in-time existence was not achieved for large initial data.

\section{Acknowledgments}

The work of the second named author is partially supported by the National Natural Science Foundation of China grants No. 11871177.

\section{References}

[1] B. Hantaek, B. Animikh, and E. Tadmor, Analyticity and decay estimates of the Navier-Stokes equations in critical Besov spaces, Arch. Ration. Mech. Anal., 205(3) (2012), 963-991.

[2] H. Bahouri, J.-Y. Chemin and R. Danchin, Fourier Analysis and Nonlinear Partial Differential Equations, volume 343 of Grundlehren der Mathematischen Wissenschaften [Fundamental Principles of Mathematical Sciences], Springer, Heidelberg, 2011.

[3] O. V. Besov, On some families of functional spaces. Imbedding and extension theorems, Dokl. Akad. Nauk SSSR, 126 (1959), 1163-1165.

[4] J.-Y. Chemin and N. Lerner. Flot de champs de vecteurs non lipschitziens et équations de Navier-Stokes, J. Differential Equations, 121(2) (1995), 314-328.

[5] J.-Y. Chemin, D.S. McCormick, J.C. Robinson, and J.L. Rodrigo, Local existence for the nonresistive MHD equations in Besov spaces, Adv. Math., 286 (2016), 1-31.

[6] X. Chen and A. Jüngel, Analysis of an incompressible Navier-Stokes-Maxwell-Stefan system, Comm. Math. Phys., 340(2) (2015), 471-497.

[7] N. Chikami and R. Danchin, On the well-posedness of the full compressible Navier-Stokes system in critical Besov spaces, J. Differential Equations, 258(10) (2015), 3435-3467.

[8] R. Duan. Green's function and large time behavior of the Navier-Stokes-Maxwell system. Anal. Appl. (Singap.), 10(2):133-197, 2012. 
[9] C.L. Fefferman, D.S. McCormick, J.C. Robinson and J.L. Rodrigo, Local existence for the non-resistive MHD equations in nearly optimal Sobolev spaces, Arch. Ration. Mech. Anal., 223(2) (2017), 677-691.

[10] P. Germain, S. Ibrahim and N. Masmoudi, Well-posedness of the Navier-Stokes-Maxwell equations, Proc. Roy. Soc. Edinburgh Sect. A, 144(1) (2014), 71-86.

[11] S. Ibrahim and S. Keraani, Global small solutions for the Navier-Stokes-Maxwell system, SIAM Journal on Mathematical Analysis, 43(5) (2011), 2275-2295.

[12] T. Kobayashi and T. Muramatu, Abstract Besov space approach to the nonstationary NavierStokes equations, Math. Methods Appl. Sci., 15(9) (1992), 599-620.

[13] H. Kozono, T. Ogawa and Y. Taniuchi, Navier-Stokes equations in the Besov space near $L^{\infty}$ and BMO, Kyushu J. Math., 57(2) (2003), 303-324.

[14] Q. Liu and Y. Su, Large time behavior for the non-isentropic Navier-Stokes-Maxwell system, Math. Methods Appl. Sci., 40(3) (2017), 663-679.

[15] T.-P. Liu, T. Yang and S.-H. Yu, Energy method for Boltzmann equation, Phys. D, 188(34)(2004), 178-192.

[16] N. Masmoudi, Global well posedness for the Maxwell-Navier-Stokes system in 2D, J. Math. Pures Appl., 93(6) (2010), 559-571.

[17] C. Miao, J. Wu and Z. Zhang, Littlewood-Paley Theory and its Applications to PDEs in Fluid Dynamics, Series on the foundation of modern mathematics, 142, Science Press, Beijing, 2012.

[18] S. Ibrahim, R.P.G. Lemarie and N. Masmoudi, Time periodic forcing and asymptotic stability for the Navier Stokes Maxwell equations, Communications on Pure and Applied Mathematics, 71(1) (2017), 51-89.

[19] Z. Tan and L. Tong, Decay estimates of solutions to the compressible Navier-Stokes-Maxwell system in $\mathbb{R}^{3}$. Commun. Math. Sci., 14(5) (2016), 1189-1212.

[20] H. Triebel, Theory of Function Spaces. II, volume 84 of Monographs in Mathematics, Birkhäuser Verlag, Basel, 1992.

[21] J. Yang and S. Wang, Convergence of compressible Navier-Stokes-Maxwell equations to incompressible Navier-Stokes equations, Sci. China Math., 57(10) (2014), 2153-2162. 